Cite this: Phys. Chem. Chem. Phys., 2014, 16, 15784

Received 16th January 2014, Accepted 11th March 2014

DOI: $10.1039 / c 4 c p 00233 d$

www.rsc.org/pccp

\section{A salt-bridge structure in solution revealed by 2D-IR spectroscopy $\dagger$}

\author{
Adriana Huerta-Viga, Sérgio R. Domingos, Saeed Amirjalayer and \\ Sander Woutersen*
}

Salt bridges are important interactions for the stability of protein conformations, but up to now it has been difficult to determine salt-bridge geometries in solution. Here we characterize the spatial structure of a salt bridge between guanidinium $\left(\mathrm{Gdm}^{+}\right)$and acetate $\left(\mathrm{Ac}^{-}\right)$using two-dimensional vibrational (2D-IR) spectroscopy. We find that as a result of salt bridge formation there is a significant change in the infrared response of $\mathrm{Gdm}^{+}$and $\mathrm{Ac}^{-}$, and cross peaks between them appear in the 2D-IR spectrum. From the 2D-IR spectrum we determine the relative orientation of the transition-dipole moments of the vibrational modes of $\mathrm{Gdm}^{+}$and $\mathrm{Ac}^{-}$, as well as the coupling between them.

Salt bridges are hydrogen-bonded ion pairs that are important for the stabilization of molecular conformations. Intermolecular salt bridges are used to create new supramolecular systems, ${ }^{1,2}$ while biological systems such as proteins often contain salt bridges that determine their structure ${ }^{3}$ and function. ${ }^{4}$ To understand the chemical functionality of salt bridges, it is essential to detect saltbridged ion pairs and to characterize their solvated structure, which is unfortunately difficult with conventional methods such as NMR. Here we report the study of a biologically-relevant ion pair formed by guanidinium $\left(\mathrm{Gdm}^{+}\right)$and acetate $\left(\mathrm{Ac}^{-}\right)$, using twodimensional infrared (2D-IR) spectroscopy. This technique is used to measure the vibrational coupling between transition-dipole moments of individual vibrations, and through a quantitative analysis of the 2D-IR spectrum, structural information can be

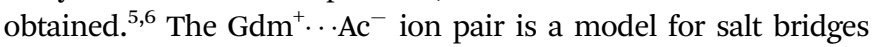
between the $\mathrm{Gdm}^{+}$side group of arginine and the carboxylate side group of glutamate or aspartate, which occur commonly in proteins. $^{7}$ The molecular structure of this ion pair is shown in Fig. 1A. Isolated $\mathrm{Gdm}^{+}$has $D_{3}$ symmetry, and has a degenerate mode due to a combined $\mathrm{CN}_{3}$ antisymmetric stretch and $\mathrm{NH}_{2}$ scissors motion at a frequency of $1600 \mathrm{~cm}^{-1}$. This degeneracy is

Van't Hoff Institute for Molecular Sciences (HIMS), University of Amsterdam, Science Park 904, Amsterdam, The Netherlands. E-mail: S.Woutersen@uva.nl; Fax: +31 2052 56456; Tel: +31 205257091

$\dagger$ Electronic supplementary information (ESI) available. See DOI: 10.1039/ c4cp00233d observed in aqueous solution, but it is broken in viscous solvents. ${ }^{9}$ When dissolving deuterated $\mathrm{Gdm}^{+}$(guanidine. $\mathrm{DCl},>98 \%$ purity, $400 \mathrm{mM}$ ) in deuterated dimethylsulfoxide (DMSO), we observe a splitting between the frequencies of the two $\mathrm{CN}_{3} \mathrm{D}_{6}{ }^{+}$modes, as can be seen in Fig. 1B. In the following, we refer to the high- and lowfrequency $\mathrm{CN}_{3} \mathrm{D}_{6}^{+}$modes of $\mathrm{Gdm}^{+}$as $\mathrm{Gdm}_{\mathrm{HF}}^{+}$and $\mathrm{Gdm}_{\mathrm{LF}}{ }^{+}$, respectively. Interestingly, when an equimolar amount of $\mathrm{Ac}^{-}$ions is added to the solution (guanidine acetate salt, $>98 \%$ purity, $400 \mathrm{mM}$ ), the splitting between $\mathrm{Gdm}_{\mathrm{HF}}{ }^{+}$and $\mathrm{Gdm}_{\mathrm{LF}}{ }^{+}$becomes larger, as seen in Fig. 1B. $\mathrm{Gdm}^{+}$and $\mathrm{Ac}^{-}$have a strong binding affinity in DMSO, forming more than $98 \%$ dimers at the concentration used in our experiments, ${ }^{10}$ which suggests that the larger splitting between the $\mathrm{Gdm}^{+}$modes is due to an interaction with the $\mathrm{Ac}^{-}$ion. Moreover, $\mathrm{Ac}^{-}$(tetrabutylammonium acetate, $>97 \%$ purity) has an absorption band at $1580 \mathrm{~cm}^{-1}$ in DMSO (shown in Fig. 1B) due to the $\mathrm{COO}^{-}$-antisymmetric-stretch mode. This mode red-shifts after dimer formation with $\mathrm{Gdm}^{+}$. The change in the infrared response of both the $\mathrm{Gdm}^{+}$and the $\mathrm{Ac}^{-}$ions upon dimer formation strongly suggests that there is a coupling between the vibrational modes of these two molecules.
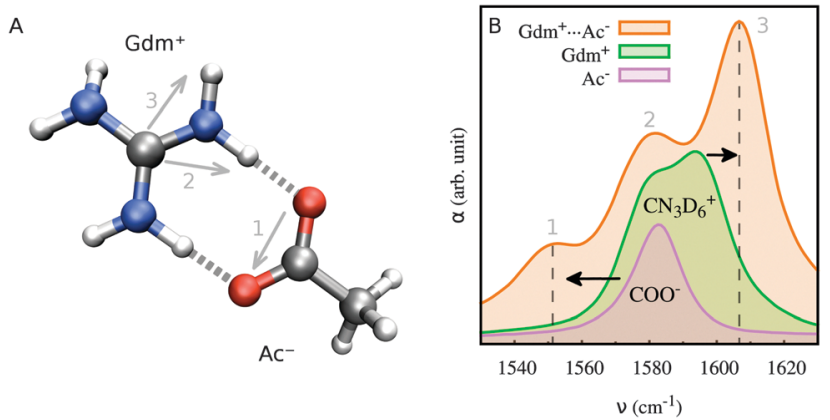

Fig. 1 (A) Molecular structure of the $\mathrm{Gdm}^{+} \ldots \mathrm{Ac}^{-}$dimer obtained using $a b$ initio methods. The corresponding transition-dipole moments of the $\mathrm{COO}^{-}$-stretch mode (1) and of the $\mathrm{CN}_{3} \mathrm{D}_{6}{ }^{+}$low- and high- frequency modes ( 2 and 3, respectively) are indicated by arrows. (B) Infrared absorption spectrum of $\mathrm{Gdm}^{+} \ldots \mathrm{Ac}^{-}, \mathrm{Gdm}^{+}$and $\mathrm{Ac}^{-}$in DMSO (solvent subtracted). Shifts of the $\mathrm{COO}^{-}$and high-frequency $\mathrm{CN}_{3} \mathrm{D}_{6}{ }^{+}$bands are indicated by arrows. 


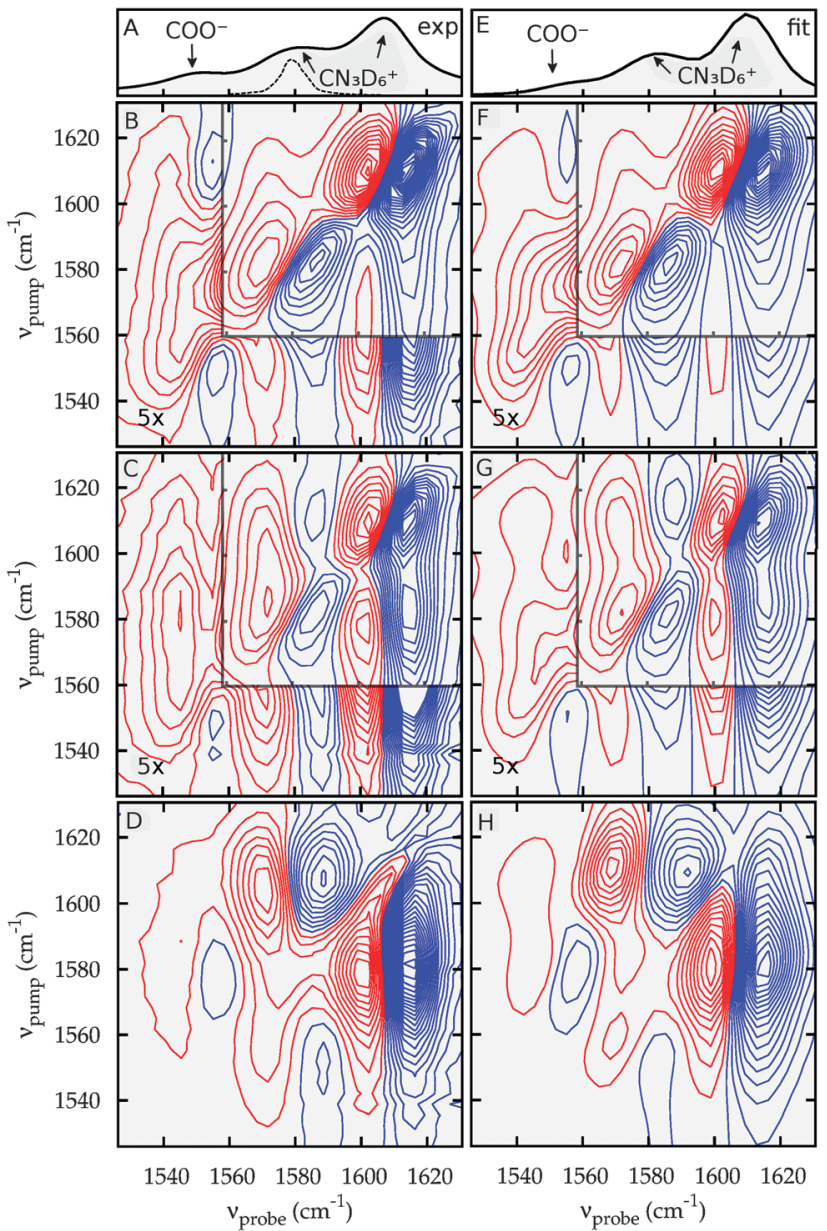

Fig. 2 (A) Infrared absorption spectrum of $\mathrm{Gdm}^{+} \ldots \mathrm{Ac}^{-}$. The dashed line indicates a representative pump-pulse spectrum. (B) and (C) 2D-IR spectra of $\mathrm{Gdm}^{+} \ldots \mathrm{Ac}^{-}$for parallel and perpendicular polarization of the pump and probe pulses, respectively (a pump-probe delay of $1.5 \mathrm{ps}$ ). Blue indicates negative absorption change, red positive absorption change. The contour intervals are $1 \mathrm{mOD}$ (parallel) and $0.5 \mathrm{mOD}$ (perpendicular), and the left and bottom parts are scaled by a factor of 5, as indicated. (D) Weighteddifference $2 \mathrm{D}-\mathrm{IR}$ spectrum $\left(3 \Delta \alpha_{\perp}-\Delta \alpha_{\|}\right)$. The contour intervals are $0.5 \mathrm{mOD}$. (E) Calculated absorption spectrum with parameters obtained from a fit to the 2D-IR spectrum. (F) and (G) Fitted 2D-IR spectra for parallel and perpendicular polarization of the pump and probe pulses, respectively. The contour intervals are the same as in $(B)$ and $(C)$. $(H)$ Weighted-difference 2D-IR spectrum that was calculated with parameters obtained from a fit to the parallel- and perpendicular-polarization spectra. Contour intervals are the same as in (D).

The 2D-IR spectra of the $\mathrm{Gdm}^{+} \cdots \mathrm{Ac}^{-}$dimer confirm unambiguously that the $\mathrm{Gdm}_{\mathrm{HF}}{ }^{+}$and $\mathrm{Gdm}_{\mathrm{LF}}{ }^{+}$modes are both coupled to the $\mathrm{COO}^{-}$-stretch mode of $\mathrm{Ac}^{-}$. To measure the 2D-IR spectra, we use a femtosecond pump-probe setup that has been described elsewhere. ${ }^{11}$ The $2 \mathrm{D}$-IR spectra are shown in Fig. 2B and $\mathrm{C}$ for parallel and perpendicular polarization of the pump and probe pulses, and weighted difference $\left(3 \Delta \alpha_{\perp}-\Delta \alpha_{\|}\right)$, respectively. The non-zero off-diagonal response (cross peaks) in the 2D-IR spectra indicates that there is a coupling between the two $\mathrm{CN}_{3} \mathrm{D}_{6}{ }^{+}$modes of $\mathrm{Gdm}^{+}$and, more importantly, between each of them and the $\mathrm{COO}^{-}$-stretch mode of $\mathrm{Ac}^{-}$. These cross peaks can be seen better in slices along both the pump and probe axes of the 2D-IR spectra. Fig. 3A shows cross sections of

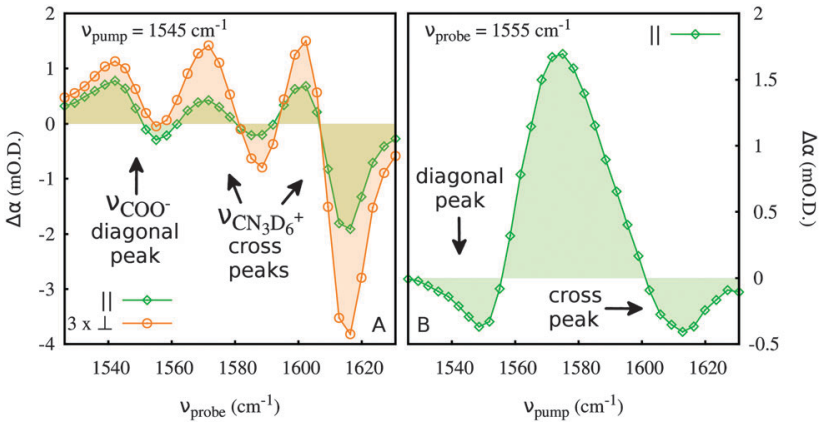

Fig. 3 (A) Cross section along the probe axis of the 2D-IR spectra for parallel and perpendicular polarization of the pump and probe pulses, and for $\nu_{\text {pump }}=\nu_{\text {coO-. }}$ (B) Cross section along the pump axis of the 2D-IR spectrum for parallel polarization of the pump and probe pulses, and for $\nu_{\text {probe }}=\nu_{\mathrm{COO}^{-}}$.

$\nu_{\mathrm{pump}}=\nu_{\mathrm{COO}^{-}}$for parallel and perpendicular polarization of the pump and probe pulses. In the cross sections, the cross peaks between the $\mathrm{COO}^{-}$-stretch mode and each of the $\mathrm{Gdm}^{+}$modes are clearly visible. Note that the diagonal signal of the $\mathrm{COO}^{-}$stretch mode has a smaller magnitude than these cross peaks because of its smaller absorption cross section (see Fig. 1B). Fig. 3B shows a cross section along the probe axis for $\nu_{\text {probe }}=$ $\nu_{\mathrm{COO}^{-}}$for parallel polarization of pump and probe pulses. The negative part at $1550 \mathrm{~cm}^{-1}$ is due to the bleaching and $\nu=1 \rightarrow 0$ stimulated emission of the $\mathrm{COO}^{-}$-stretch mode on the diagonal of the $2 \mathrm{D}$ spectrum. The positive region centered at $1580 \mathrm{~cm}^{-1}$ is the low-probe-frequency tail of the excited-state absorption of the $\mathrm{Gdm}_{\mathrm{LF}}^{+}$mode on the diagonal. The negative region at $1610 \mathrm{~cm}^{-1}$ is the negative part of the cross peak between the $\mathrm{COO}^{-}$-stretch mode and the $\mathrm{Gdm}_{\mathrm{HF}}{ }^{+}$mode. Note that the cross peaks between $\mathrm{Gdm}_{\mathrm{HF}}{ }^{+}$and $\mathrm{Ac}^{-}$are more clearly visible in the parallel-polarization 2D-IR spectrum (Fig. 2B), and almost vanish in the $3 \Delta \alpha_{\perp}-\Delta \alpha_{\|}$difference spectrum (Fig. 2D). This behaviour indicates that the transition-dipole moments of these modes are approximately parallel. ${ }^{12}$ In contrast, the cross peaks between $\mathrm{Gdm}_{\mathrm{LF}}{ }^{+}$and $\mathrm{Ac}^{-}$appear clearly in the $3 \Delta \alpha_{\perp}-\Delta \alpha_{\|}$difference spectrum (Fig. 2D), which suggests that their transition-dipole moments are at a nonzero angle. ${ }^{12}$

In order to obtain structural information from the 2D-IR spectra, we use a vibrational-exciton model (see ESI $\dagger$ for a description of the model). ${ }^{12}$ It has been shown before that in addition to coupling between the two $\mathrm{CN}_{3} \mathrm{D}_{6}{ }^{+}$modes, there is also energy transfer between them, ${ }^{9}$ but in DMSO this process is slow enough to be neglected at the delay at which we measured the 2D-IR spectra used for the structural analysis (1.5 ps, see ESI $\dagger$ ). We determine the spectral and structural parameters from a global fit to the parallel and perpendicular 2D-IR spectra. Fig. $2 \mathrm{~F}$ and $\mathrm{G}$ show the fitted 2D-IR spectra, which are in very good agreement with the measured ones. Note that the difference spectrum, shown in Fig. 2D is not fitted but the corresponding calculated spectrum, shown in Fig. $2 \mathrm{H}$, is also in very good agreement with the measurement. We find that in order to obtain a good fit, an inhomogeneity in the coupling between $\mathrm{Gdm}_{\mathrm{LF}}{ }^{+}$and $\mathrm{Gdm}_{\mathrm{HF}}{ }^{+}$modes has to be taken into account in the model. 
Table 1 Coupling $\beta$ and angle $\theta$ between the transition-dipole moments of the $\mathrm{COO}^{-}$-stretch mode of $\mathrm{Ac}^{-}$and the two $\mathrm{CN}_{3} \mathrm{D}_{6}{ }^{+}$modes of $\mathrm{Gdm}^{+}$ as obtained from the fit $\left(\chi_{r}^{2}=41\right)$. See ESI for estimates of the confidence limits

\begin{tabular}{lcr}
\hline Modes & $\beta\left(\mathrm{cm}^{-1}\right)$ & $\theta$ \\
\hline $\mathrm{Gdm}_{\mathrm{HF}}{ }^{+}-\mathrm{Gdm}_{\mathrm{LF}}{ }^{+}$ & -8 & $105^{\circ}$ \\
$\mathrm{Gdm}_{\mathrm{HF}}{ }^{+}-\mathrm{Ac}^{-}$ & -11 & $180^{\circ}$ \\
$\mathrm{Gdm}_{\mathrm{LF}}{ }^{-}-\mathrm{Ac}^{-}$ & 8 & $75^{\circ}$
\end{tabular}

The couplings between the three modes involved in the salt bridge $\left(\mathrm{Gdm}_{\mathrm{HF}}^{+}, \mathrm{Gdm}_{\mathrm{LF}}{ }^{+}\right.$and $\left.\mathrm{Ac}^{-}\right)$, and the angles between their transition-dipole moments were parameters of the fit and are listed in Table 1 (see $\mathrm{ESI} \dagger$ for a complete list of fit parameters). The coupling between $\mathrm{Gdm}_{\mathrm{HF}}{ }^{+}$and $\mathrm{Gdm}_{\mathrm{LF}}{ }^{+}$modes, which involve oscillations of the same $\mathrm{CN}$ and $\mathrm{NH}$ bonds, is $-8 \mathrm{~cm}^{-1}$. Remarkably, the couplings between the $\mathrm{COO}^{-}$-stretch mode and the $\mathrm{Gdm}_{\mathrm{HF}, \mathrm{LF}}{ }^{+}$modes are of a similar magnitude, $(-11$ and $8 \mathrm{~cm}^{-1}$, respectively) even though these vibrational modes share neither atoms nor bond: these couplings originate purely from the salt bridge between the two molecules. The large couplings between the $\mathrm{COO}^{-}$and the $\mathrm{Gdm}_{\mathrm{HF}, \mathrm{LF}}{ }^{+}$modes show that the frequency shifts observed in the IR spectrum upon salt-bridge formation (Fig. 1A) are mostly due to splitting of the coupled modes rather than due to a change in the local-mode frequencies. This is confirmed by the fact that the $\mathrm{COO}^{-}$peak shifts much less when acetate forms a salt bridge with protonated $\mathrm{Gdm}^{+}$, in which the two $\mathrm{CN}_{3} \mathrm{H}_{6}{ }^{+}$modes are at a higher frequency (see ESI $\dagger$ ). The angles between the transition-dipole moments of the salt-bridged vibrational modes are in good agreement with the planar geometry shown in Fig. 1A. The planarity of a salt bridge is often taken as a metric of its quality in X-ray studies, ${ }^{13}$ and our results suggest that in DMSO solution, the geometry of an isolated salt bridge, in which steric constraints are absent, is indeed planar. This finding is also in agreement with experimental results in peptides in the gas phase, where planar geometries for salt bridges between $\mathrm{Arg}^{+}$and $\mathrm{Glu}^{-}$were found. ${ }^{14,15}$ We have performed complementary ab initio calculations on the $\mathrm{Gdm}^{+} \cdots \mathrm{Ac}^{-}$dimer using Gaussian $03^{16}$ at the MP2/ $6-311+G(d)$ level of theory. The calculation predicts a planar geometry for the salt bridge, i.e., the three transition-dipole moments are coplanar, as shown in Fig. 1A. The angle between the transition-dipole vectors of the two $\mathrm{Gdm}^{+}$modes is smaller than for isolated guanidinium, ${ }^{9}$ most likely as a result of salt bridge formation with the $\mathrm{Ac}^{-}$ion. We find in the calculation that the $\mathrm{Gdm}_{\mathrm{HF}}^{+}$mode is antisymmetric with respect to the symmetry axis through the $\mathrm{C}-\mathrm{C}$ bond of $\mathrm{Ac}^{-}$, which explains the large coupling with the likewise antisymmetric $\mathrm{COO}^{-}$-stretch mode. ${ }^{17}$ The $\mathrm{Gdm}_{\mathrm{LF}}{ }^{+}$mode is symmetric with respect to this symmetry axis, so it is remarkable that it couples quite strongly to the $\mathrm{COO}^{-}$-stretch mode despite their different symmetry. The infrared-absorption bands predicted from the $a b$ initio calculations also show a shift of the $\mathrm{Gdm}^{+}$modes upon salt bridge formation, in agreement with our measurements (see ESI $\dagger$ ).

In conclusion, we were able to detect the existence of a salt bridge between $\mathrm{Gdm}^{+}$and $\mathrm{Ac}^{-}$in solution using 2D-IR spectroscopy. The method we present is also applicable for the detection of salt bridges between arginine and glutamate or aspartate in peptides and proteins. Spectral crowding due to the broadening of the amide I band in larger systems can be avoided by using isotopic labels: ${ }^{15} \mathrm{~N}$ for $\mathrm{Arg}^{+}$results in $\sim 40 \mathrm{~cm}^{-1}$ redshift, ${ }^{18}$ and ${ }^{18} \mathrm{O}$ for $\mathrm{Glu}^{-}$results in $\sim 10 \mathrm{~cm}^{-1}$ redshift. ${ }^{12}$ We characterize the coupling between two $\mathrm{CN}_{3} \mathrm{D}_{6}{ }^{+}$ modes of $\mathrm{Gdm}^{+}$and the $\mathrm{COO}^{-}$-stretch mode of $\mathrm{Ac}^{-}$, and we find that the $\mathrm{COO}^{-}$-stretch mode couples more strongly to the high-frequency $\mathrm{CN}_{3} \mathrm{D}_{6}{ }^{+}$mode than to the low-frequency one, most likely because of their similar symmetry. Our data indicate a salt-bridge geometry in which the $\mathrm{Gdm}^{+}$and $\mathrm{COO}^{-}$moieties are coplanar. Our results show that 2D-IR spectroscopy is a method of wide applicability to detect and determine the geometry of salt bridges in solution, with picosecond temporal resolution.

\section{References}

1 B. Kuberski and A. Szumna, Chem. Commun., 2009, 1959-1961.

2 L. E. R. O'Leary, J. A. Fallas, E. L. Bakota, M. K. Kang and J. D. Hartgerink, Nature Chem., 2011, 3, 821-828.

3 S. Kumar and R. Nussinov, J. Mol. Biol., 1999, 293, 1241-1255.

4 J. M. Christie, A. S. Arvai, K. J. Baxter, M. Heilmann, A. J. Pratt, A. O'Hara, S. M. Kelly, M. Hothorn, B. O. Smith, K. Hitomi, G. I. Jenkins and E. D. Getzoff, Science, 2012, 335, 1492-1496.

5 O. Golonzka and A. Tokmakoff, J. Chem. Phys., 2001, 115, 297.

6 M. T. Zanni, N. H. Ge, Y. S. Kim and R. M. Hochstrasser, Proc. Natl. Acad. Sci. U. S. A., 2001, 98, 11265-11270.

7 K. D. Walker, T. P. Causgrove and R. T. Sauer, J. Mol. Model., 2009, 15, 1213-1219.

8 R. J. Sension and B. Hudson, J. Phys. Chem., 1990, 94, 4015-4025.

9 D. Y. Vorobyev, C.-H. Kuo, D. G. Kuroda, J. N. Scott, J. M. Vanderkooi and R. M. Hochstrasser, J. Phys. Chem. B, 2010, 114, 2944-2953.

10 B. Linton and A. D. Hamilton, Tetrahedron, 1999, 55, 6027-6038.

11 A. Huerta-Viga, D. J. Shaw and S. Woutersen, J. Phys. Chem. B, 2010, 114, 15212-15220.

12 P. Hamm and M. Zanni, Concepts and Methods of $2 D$ Infrared Spectroscopy, Cambridge University Press, 1st edn, 2011.

13 J. E. Donald, D. W. Kulp and W. F. DeGrado, Proteins, 2011, 79, 898-915.

14 A. M. Rijs, G. Ohanessian, J. Oomens, G. Meijer, G. von Helden and I. Compagnon, Angew. Chem., Int. Ed., 2010, 49, 2332-2335.

15 S. Jaeqx, J. Oomens and A. M. Rijs, Phys. Chem. Chem. Phys., 2013, 15, 16341-16352.

16 M. J. Frisch, et al., Gaussian 03, Revision C.02, Gaussian, Inc., Wallingford, CT, 2004.

17 B. Sharma, Spectroscopy, Krishna Prakashan, 1981.

18 I. T. Arkin, Curr. Opin. Chem. Biol., 2006, 10, 394-401. 